\title{
Representação de um projeto de sistema de esgoto em um software BIM
}

\author{
Representation of a sewage system project in BIM software \\ Representación de un proyecto de alcantarillado en software BIM
}

Recebido: 05/11/2021 | Revisado: 12/11/2021 | Aceito: 16/11/2021 | Publicado: 27/11/2021

\author{
Emmanuel Kennedy da Costa Teixeira \\ ORCID: https://orcid.org/0000-0001-7598-0240 \\ Universidade Federal de São João del-Rei, Brasil \\ E-mail: emmanuel.teixeira@ufsj.edu.br \\ André Luiz Santos Ribeiro \\ ORCID: https://orcid.org/0000-0002-7981-9240 \\ Universidade Federal de São João del-Rei, Brasil \\ E-mail: andreluizsantosribeiro@ hotmail.com \\ Leonardo Montes Furtado \\ ORCID: https://orcid.org/0000-0003-0924-3669 \\ Universidade Federal de São João del-Rei, Brasil \\ E-mail: leo-montes@ hotmail.com
}

\begin{abstract}
Resumo
Para dimensionar e executar uma rede coletora de esgoto há muitos desafios, sendo o principal deles a compatibilização entre as outras redes existentes na rua. Para minimizar esse problema, a metodologia Building Information Modeling (BIM) pode auxiliar, visto que ela é capaz de gerar um modelo virtual equivalente ao real que pode ser visualizado em 3D e simulado. Embora essa tecnologia esteja disponível no mercado há anos, ela ainda é incipiente quando aplicada em projetos de infraestruturas. O presente trabalho teve como objetivo representar um projeto de esgotamento sanitário por meio do software Civil 3D, que utiliza a metodologia BIM, onde foram detalhados os procedimentos para criação da rede e gerados o perfil longitudinal, o quantitativo e o descritivo das estruturas. A utilização do programa foi extremamente eficiente para representação e compatibilização de projetos de infraestruturas.
\end{abstract}

Palavras-chave: Infraestrutura; Civil 3D; Saneamento.

\begin{abstract}
There are many challenges to dimension and implement a sewage collection network, the main one being the compatibility with the other networks existing on the street. To minimize this problem, the Building Information Modeling (BIM) methodology can help, as it is capable of generating a virtual model equivalent to the real one that can be visualized in 3D and simulated. Although this technology has been available on the market for years, it is still in its infancy when applied to infrastructure projects. The present work aimed to represent a sanitary sewage project through the Civil 3D software, which uses the BIM methodology, where the procedures for the creation of the network were detailed and the longitudinal, quantitative and descriptive profiles of the structures were generated. The use of the program was extremely efficient for the representation and compatibility of infrastructure projects.
\end{abstract}

Keywords: Infrastructure; Civil 3D; Sanitation.

\section{Resumen}

Son muchos los desafíos para dimensionar e implementar una red de recolección de aguas residuales, siendo el principal la compatibilidad con las otras redes existentes en la calle. Para minimizar este problema, la metodología Building Information Modeling (BIM) puede ayudar, ya que es capaz de generar un modelo virtual equivalente al real que se puede visualizar en 3D y simular. Aunque esta tecnología ha estado disponible en el mercado durante años, todavía está en pañales cuando se aplica a proyectos de infraestructura. El presente trabajo tuvo como objetivo representar un proyecto de alcantarillado sanitario a través del software Civil 3D, que utiliza la metodología BIM, donde se detallaron los procedimientos para la creación de la red y se generaron los perfiles longitudinales, cuantitativos y descriptivos de las estructuras. El uso del programa fue extremadamente eficiente para la representación y compatibilidad de proyectos de infraestructura.

Palabras clave: Infraestructura; Civil 3D; Saneamiento.

\section{Introdução}

O saneamento básico é um direito assegurado pela Constituição brasileira. Entretanto, com o crescimento 
desordenado das cidades é cada vez mais necessário que sejam realizados estudos sobre sistemas de esgotamento sanitário, a fim de garantir uma qualidade de vida para a população. O sistema de coleta e de tratamento do esgoto é definido como a gestão das condições ambientais e a prestação de serviços para prevenir e controlar a propagação de doenças e melhorar o bem-estar das populações humanas (Maranhão, 2021), além de minimizar problemas com odores e garantir a qualidade dos recursos hídricos e do abastecimento de água. Segundo Rodrigues et al. (2020), a crescente urbanização das cidades vem fazendo com que as concessionárias de saneamento e os órgãos governamentais adequem e aprimorem os sistemas, seja por meio da implantação de novas redes ou pela expansão das já existentes.

O dimensionamento de uma rede coletora deve seguir parâmetros racionais e otimizados, certificando a diminuição de impactos econômicos e socioambientais. Mesmo com o auxílio da NBR 9649/1986 há muito desafios durante a execução desse tipo de projeto, dentre eles está a dificuldade de compatibilizar a rede de esgoto com as outras redes existentes na rua, como drenagem, distribuição de água, gás, energia e telefonia. No Brasil, tradicionalmente se utilizam ferramentas capazes de representar os projetos apenas em 2D, o que gera uma representação planificada do que será construído e não permite uma análise conjunta das redes. Entretanto, nos últimos anos, alguns projetos na área de saneamento têm utilizado softwares que representam o projeto em 3D. Além disso, tem-se avançado na área a utilização da metodologia Building Information Modeling (BIM), a qual integra Arquitetos, Engenheiros e Construtores (AEC) ao gerenciamento digital do projeto e seus dados ao longo da construção (Eastman et al., 2008; Smith, 2014; Maína, 2018).

$\mathrm{Na}$ metodologia BIM, as informações do projeto se encontram interligadas e processadas em tempo real, o que diminui a chance de propagação de erros e maior eficiência na questão econômica (Li et al., 2018; Santos \& Piacente, 2021). Segundo Carmona (2017), essas três letras carregam um significado muito maior; elas representam toda uma metodologia de trabalho e tem proporcionado mudanças na área de engenharia de construção (Gomes et al., 2018). O modelo virtual é equivalente ao real, sendo que ele pode ser visualizado em 3D e simulado a fim de entender o seu comportamento. A metodologia permite a integração entre os projetos e se ter um ambiente único para todos os projetistas (Salomão et al., 2019), de forma que é possível testar soluções para problemas de compatibilização (Coutinho et al., 2021), otimizando recursos e processos, além de proporcionar uma comunicação mais eficiente entre os participantes do projeto.

A metodologia BIM está disponível no mercado há anos, sendo que no Brasil houve uma grande adoção entre 2014 e 2016, devido à Copa do Mundo e aos Jogos Olímpicos (Santos et al., 2021), porém ainda podem ser considerada nova na indústria brasileira na área da Arquitetura, Engenharia e Construção (Cardoso et al., 2021) e incipiente no setor de saneamento (Barbosa, 2017). Dessa forma, há ainda muitos desafios para a sua implementação (Checcucci, 2019). Bradley et al. (2016) enfatizam que há um baixo número de pesquisas que utilizam o BIM na área de infraestrutura. Atualmente ainda é notória a escassez de estudos voltados para o projeto de rede coletora de esgoto em BIM. Na maioria das vezes, o que se tem é a aplicação dessa tecnologia para o projeto da estação de tratamento de esgoto (ETE), mas não para o da rede coletora. Torres (2020) realizou um trabalho focado em obras de infraestrutura, especialmente em obras de drenagem pluvial e esgoto onde o BIM ainda precisa ser mais explorado. Neste estudo, o autor realizou uma análise em uma obra de infraestrutura urbana no Equador, a partir da qual ele elaborou um fluxo de trabalho como uma alternativa para melhorar o planejamento da obra com o uso do BIM. Edmondson et al. (2018) desenvolveram um modelo de informação de ativos para uma rede de drenagem existente. Os autores descreveram uma abordagem de análise dados para facilitar a previsão em tempo real de inundações.

Como no Brasil o BIM ainda é pouco utilizado para projeto de rede de esgoto, as empresas que atuam nesta área ainda não estão preparadas para essa mudança. Por isso, há uma dificuldade muito grande de encontrar catálogos de materiais e estruturas de rede de esgoto, que sejam compatíveis com a metodologia, ao contrário que para os projetos em 2D os catálogos são obtidos facilmente. Além disso, na literatura há escassez de trabalhos que auxiliem na utilização de programas BIM para a representação de sistema de esgoto. Segundo Correa et al. (2019) ainda há muito a ser desenvolvido para o BIM voltado para a 
área de infraestrutura, seja uma evolução dos softwares de modelagem, planejamento e gestão, estabelecimento de diretrizes claras para contratação e fiscalização, e evolução para novas utilizações do modelo de infraestrutura.

Assim, o principal objetivo deste trabalho foi representar um projeto de rede de esgoto em um software BIM. Dessa forma, pôde-se ter uma completa noção do projeto antes da execução.

\section{Metodologia}

\section{1 Área de estudo}

Neste trabalho, foi modelado um projeto existente, o qual é de um sistema de esgoto de um loteamento com 118 lotes e com uma área total de 20 ha. A modelagem do terreno em estudo pode ser analisada na Figura 1.

Figura 1 - Modelagem do terreno onde será executado o sistema de esgoto.

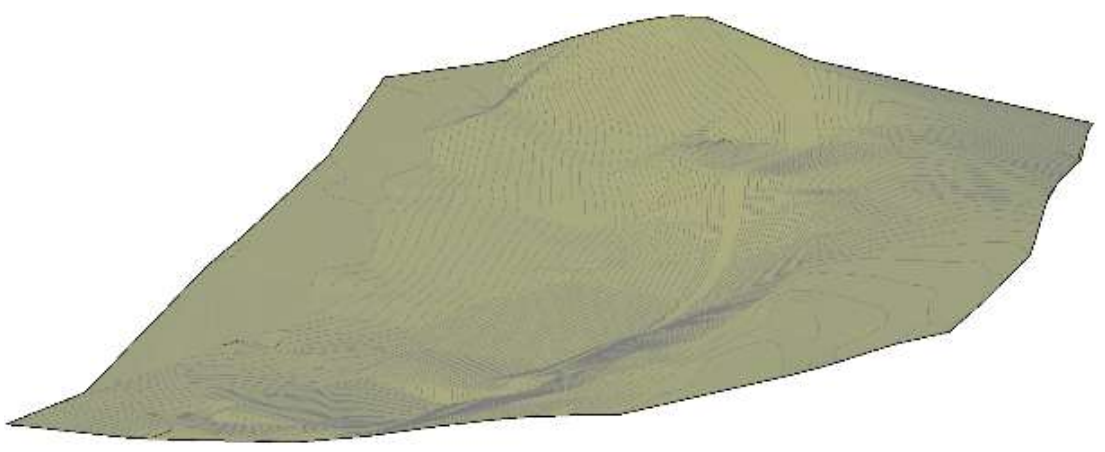

Fonte: Autores.

A representação da rede traçada, ilustrada na Figura 2, aproveita ao máximo as curvas de níveis do terreno garantindo a redução da profundidade e, consequentemente, a diminuição dos gastos por escavação e materiais. Neste projeto, parte da vazão foi atendida por uma fossa e a maior parte foi escoada até a ETE. O projeto adotou, majoritariamente, redes simples ou com contribuição bilateral para as ruas do traçado, sendo a rede executada no terço da via mais próximo ao lado em que os lotes possuem soleiras mais baixas, favorecendo o atendimento.

A tubulação implementada possui 1054,10 m, sendo composta por 16 trechos. O material escolhido no projeto foi o policloreto de vinil (PVC) liso com junta elástica e anel de vedação de borracha. Dentre as estruturas presente na rede, o projeto possui três terminais de inspeção e limpeza (TIL). Ademais, há 15 poços de visitas (PV) devido à necessidade de mudança de direção, declividade, diâmetro e de unir coletores. Devido ao custo inferior, facilidade de obtenção e opções de profundidade, o projeto adotou o concreto como material dos PVs. 
Figura 2 - Traçado da rede de esgoto.

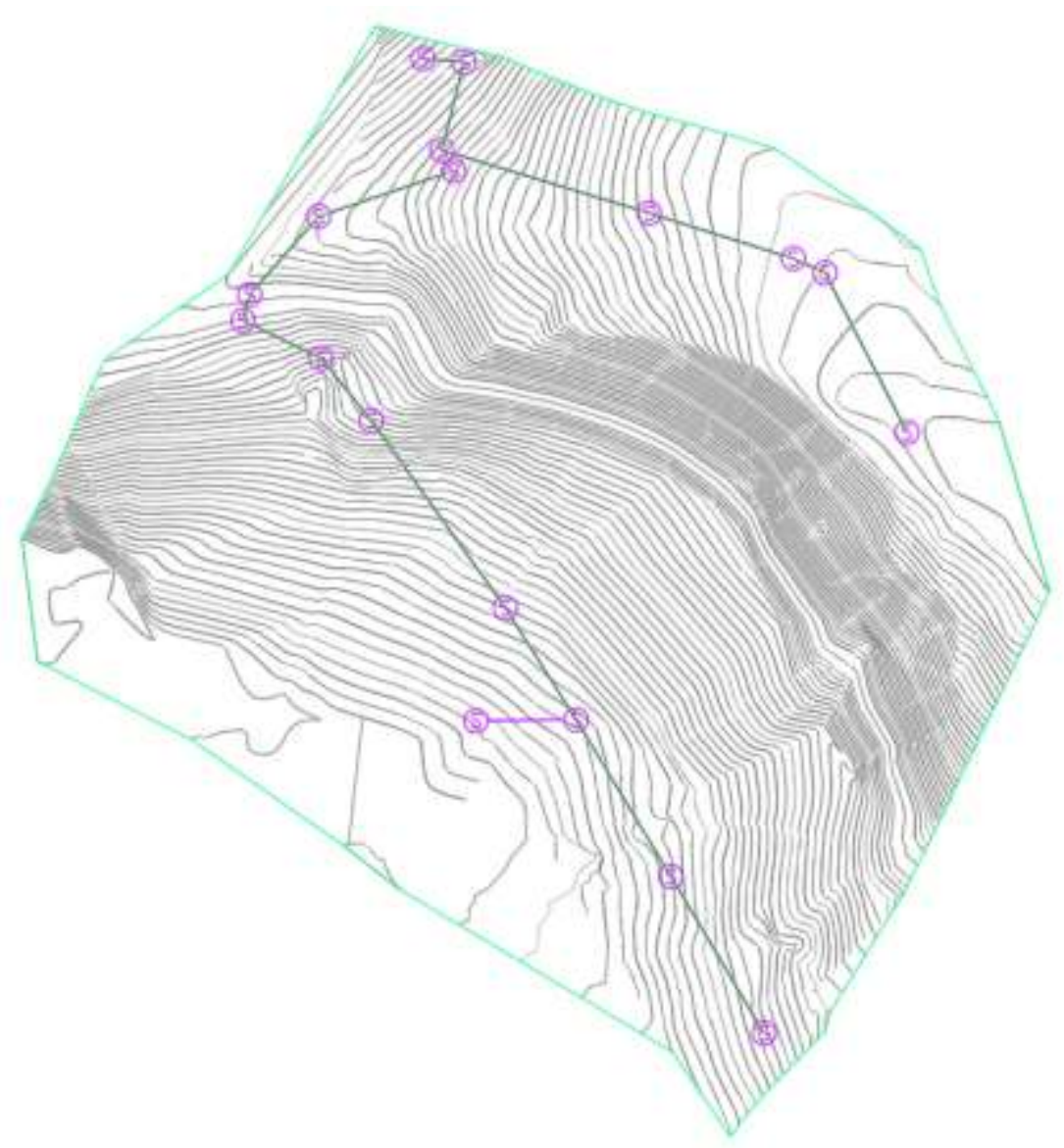

Fonte: Autores.

\subsection{Modelagem do projeto do sistema de esgoto}

O projeto do sistema de esgoto é componente das obras de infraestruturas, as quais podem ter mais qualidades quando desenvolvidas com o BIM (Costin et al., 2018). Assim como Augustine e Eldhose (2016), Kim et al. (2014), Huang et al. (2011), entre outros, a metodologia BIM foi aplicada para um projeto de infraestrutura. Entretanto, diferentemente dos autores citados, neste trabalho foi realizado um estudo de caso representado em ambiente BIM o projeto de um sistema de esgoto. Para isso, foi utilizado o software Civil 3D, o qual é capaz de trabalhar com objetos paramétricos que dispensam a necessidade de desenhos manuais. Além disso, ele permite extrair informações volumétricas, realizar análises sobre o modelo projetado e extração da documentação do projeto, reforça Cardoso (2020). Diferentemente do AutoCAD, ele trabalha com modelo dinâmico do projeto e possui objetos distintos como superfícies, alinhamentos e corredores.

Inicialmente, adequou-se o software com os parâmetros da região onde ele será utilizado. Para isso, instalou-se o pacote Country Kit Brazil, o qual foi obtido no site de suporte do Civil 3D.

Com o programa regionalizado, criou-se a superfície do terreno referente ao projeto. Para isso, seguiram-se os seguintes passos:

i. $\quad \mathrm{O}$ arquivo dwg com as curvas de níveis foi aberto;

ii. Home $>$ Surfaces $>$ Create Surface;

iii. $\quad$ Toolspace $>$ Surfaces $>$ Superficie Criada $>$ Definition $>$ Contours $>$ Add .

Para criar o traçado da rede coletora foram realizadas as seguintes ações:

i. O traçado da rede foi realizado com a ferramenta Polyline; 


\section{ii. $\quad$ Home $>$ Pipe Network > Create Pipe Network from Object $>$ Select Object.}

\subsection{Automatização dos perfis longitudinais e quantitativos}

Uma das principais vantagens da utilização do Civil 3D foi a geração automática dos perfis longitudinais e de tabelas com detalhes e quantitativos das estruturas. Para obter o perfil longitudinal de um trecho foram realizados os seguintes passos:

i. Um alinhamento no traçado da rede coletora foi criado: Home > Aligment $>$ Aligment Creation Tools;

ii. $\quad$ Home $>$ Profile $>$ Create Surface Profile $>$ Add $>$ Draw in profile view $>$ Create Profile View.

Com os perfis longitudinais criados, pôde-se facilmente editar as estruturas, adequando-as ao dimensionamento da rede. Para isso, a estrutura desejada foi selecionada e as seguintes ferramentas foram utilizadas: Home > Pipe Properties/Structure Properties.

Para gerar as tabelas com os detalhes das estruturas as ferramentas utilizadas foram:

Annotate > Add Tables > Pipe Network > Add StructurelAdd Pipe.

\section{Resultados e Discussão}

\subsection{Modelagem do projeto do sistema de esgoto}

Com a rede representada no Autodesk Civil 3D, foi possível observar os rótulos com o detalhamento das estruturas no traçado da rede do sistema de esgoto. Além disso, pôde-se visualizar a modelagem do projeto aproximando muito da realidade da construção. Assim, futuramente será possível para o projetista analisar e compatibilizar a obra com as outras construções existentes.

Na Figura 3 consta uma parte da ilustração do traçado da rede de esgoto realizado no software. Este trecho possui seis tubos que estão ligados por sete PVs (PV1 ao PV7) e o destino final, o qual é uma fossa séptica.

Figura 3 - Traçado da rede de esgoto do trecho 1.

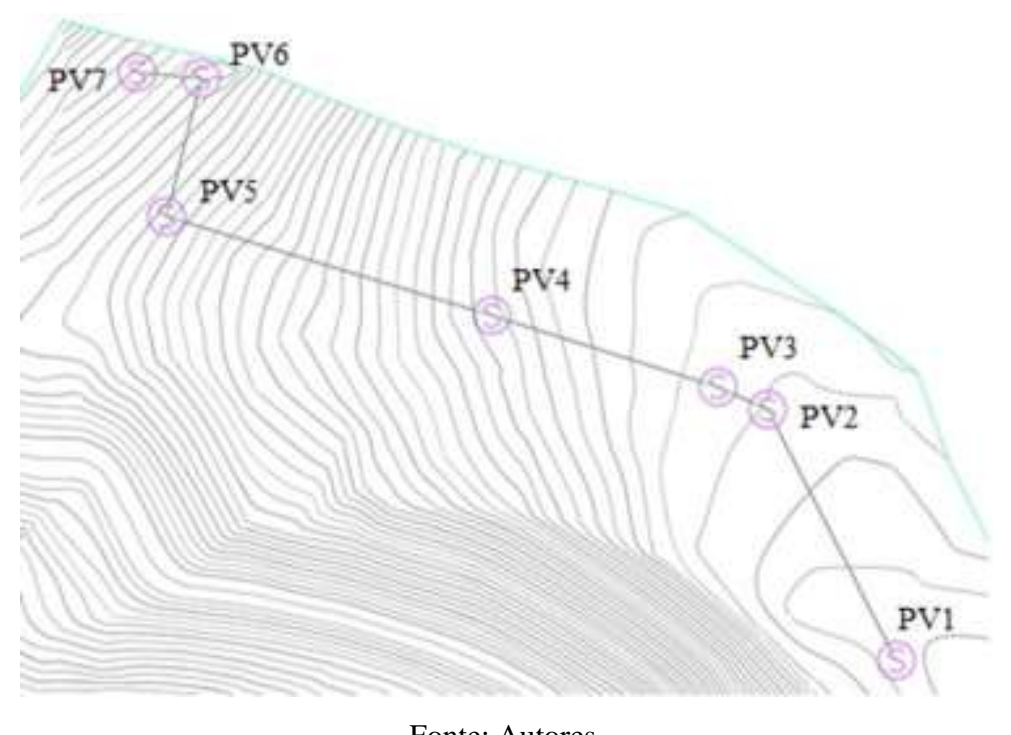

Fonte: Autores.

Na Figura 4, está ilustrada a representação da modelagem em 3D do trecho 1, sendo que o PV mais à direita (PV1) se encontra em uma cota superior aos demais PVs, aproveitando, assim, o greide do terreno. 
Research, Society and Development, v. 10, n. 15, e343101522860, 2021

(CC BY 4.0) | ISSN 2525-3409 | DOI: http://dx.doi.org/10.33448/rsd-v10i15.22860

Figura 4 - Modelagem 3D do trecho 1 da rede de esgoto.

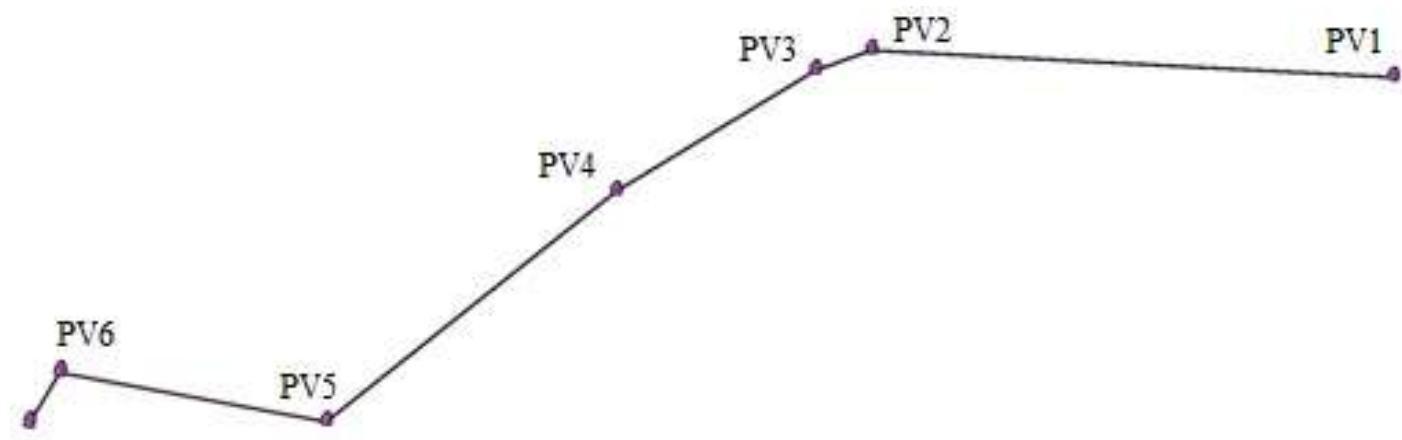

PV7

Fonte: Autores.

Na Figura 5 é observado o traçado do segundo trecho da rede coletora de esgoto, o qual é composto por dez tubos e onze PVs (PV8 ao PV18), sendo esse trecho destinado a uma ETE localizada após o PV18.

Figura 5 - Traçado de rede de esgoto do trecho 2.

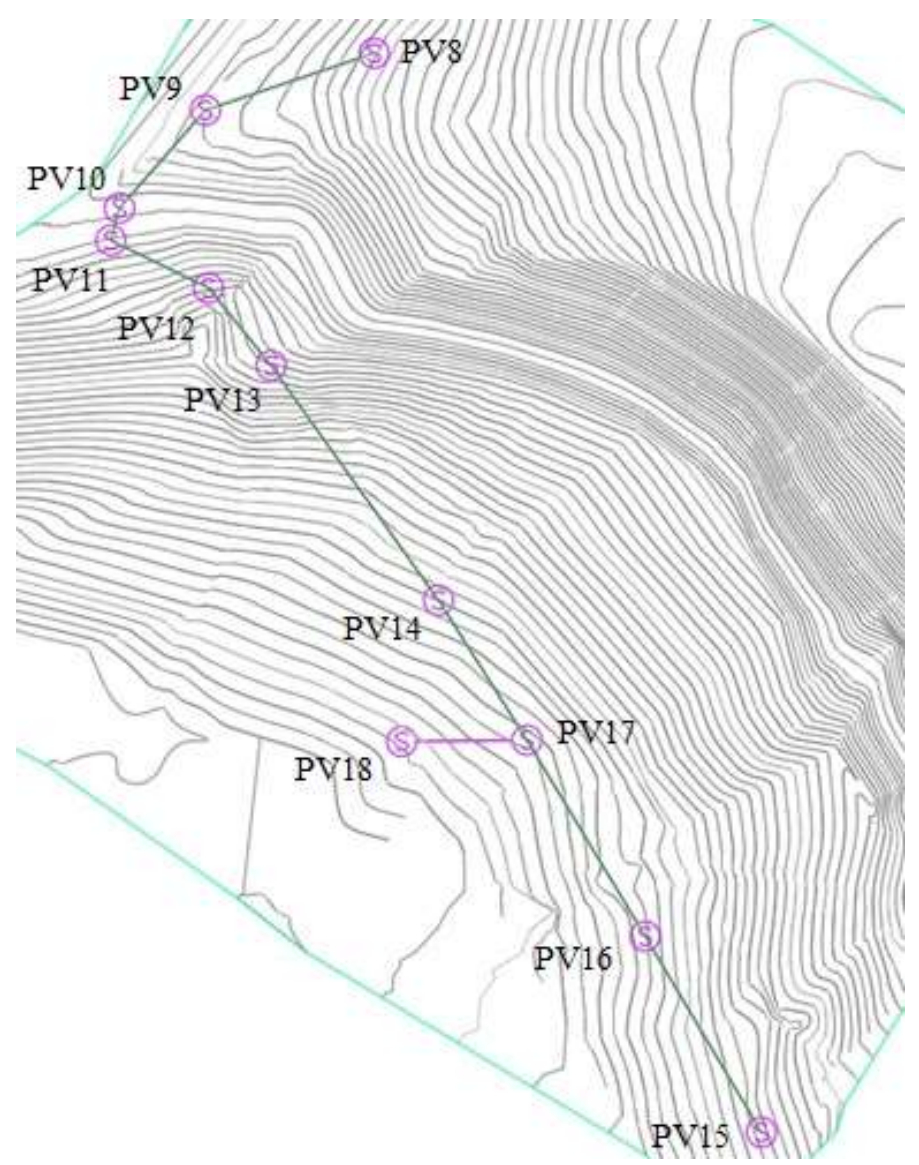

Fonte: Autores.

A modelagem em 3D desse mesmo trecho pode ser observada na Figura 6. Nela, tem-se que o PV8 e o PV15, os quais são os da extremidade, convergem seu fluxo para o PV17, o qual direciona o esgoto para o PV18. 
Figura 6 - Modelagem 3D do trecho 2.

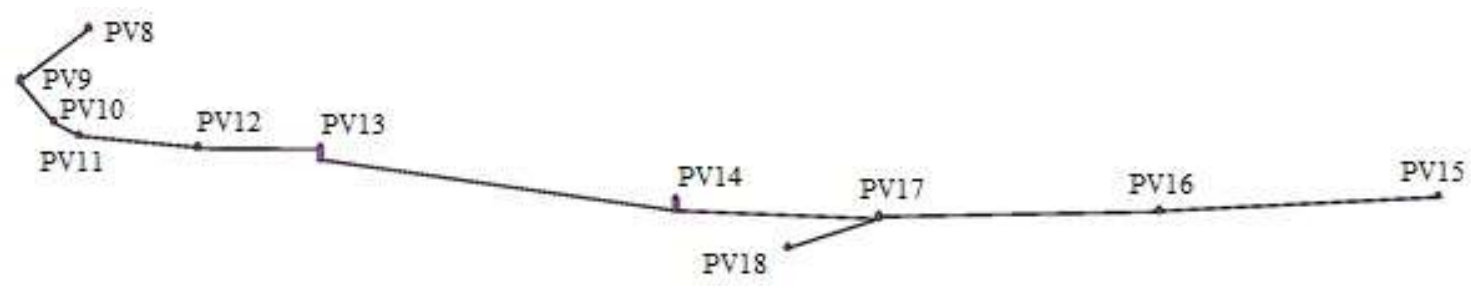

Fonte: Autores.

A ilustração da modelagem da rede coletora de esgoto e do terreno pode ser observada na Figura 7. Na Figura 8, está representada a modelagem dos PVs.

Figura 7 - Modelagem de um fragmento da rede coletora de esgoto.

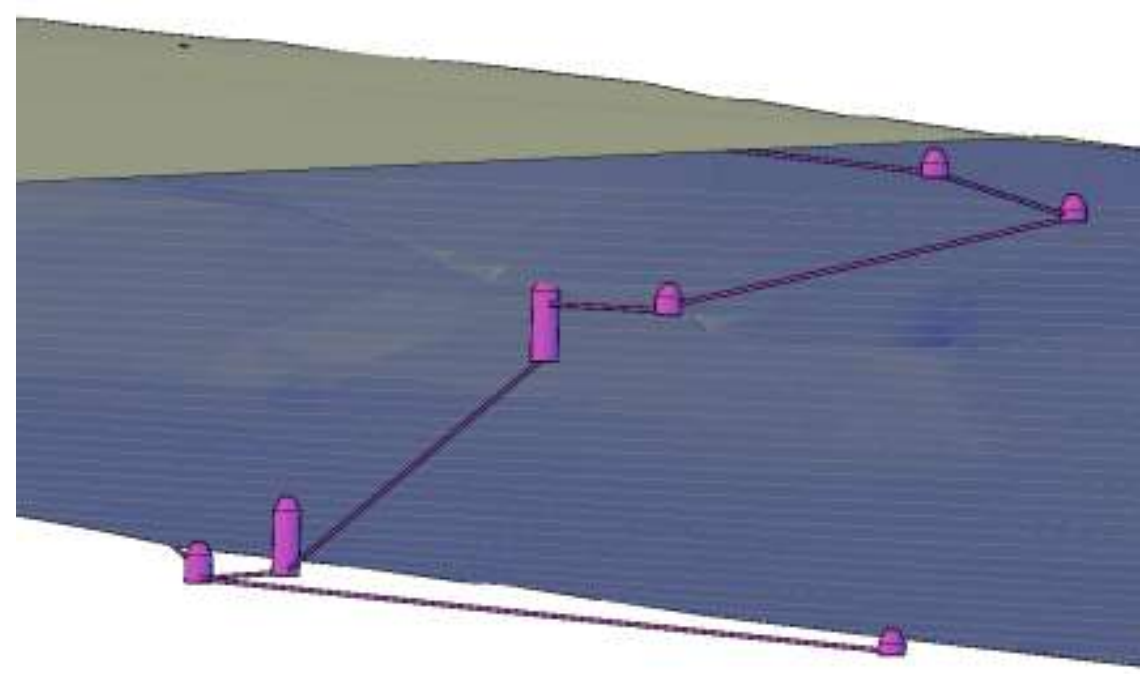

Fonte: Autores.

Figura 8 - Representação de um PV no Civil 3D.

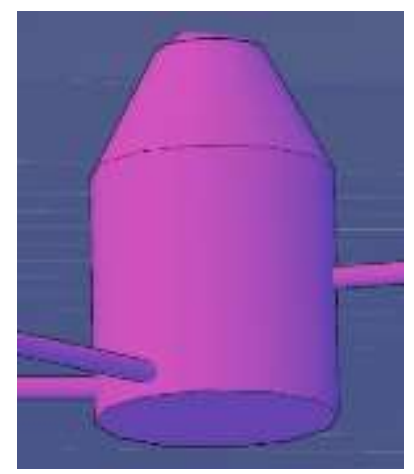

Fonte: Autores.

\subsection{Automatização dos perfis longitudinais e quantitativos}

Dentre as diversas vantagens da tecnologia BIM, a que se destaca para projetos de saneamento é a automatização dos perfis longitudinais, os quais são gerados pelo próprio Civil 3D. Salienta-se ainda a capacidade do programa de fornecer tabelas com detalhes das estruturas presentes e o quantitativo de cada material utilizado no projeto. Na Figura 9, pode-se observar o perfil longitudinal do trecho 1, o qual foi gerado automaticamente pelo software. Nota-se que o perfil do terreno 
está representado pelo traçado vermelho e as estruturas da rede em roxo.

Figura 9 - Perfil longitudinal do trecho 1 da rede coletora de esgoto.

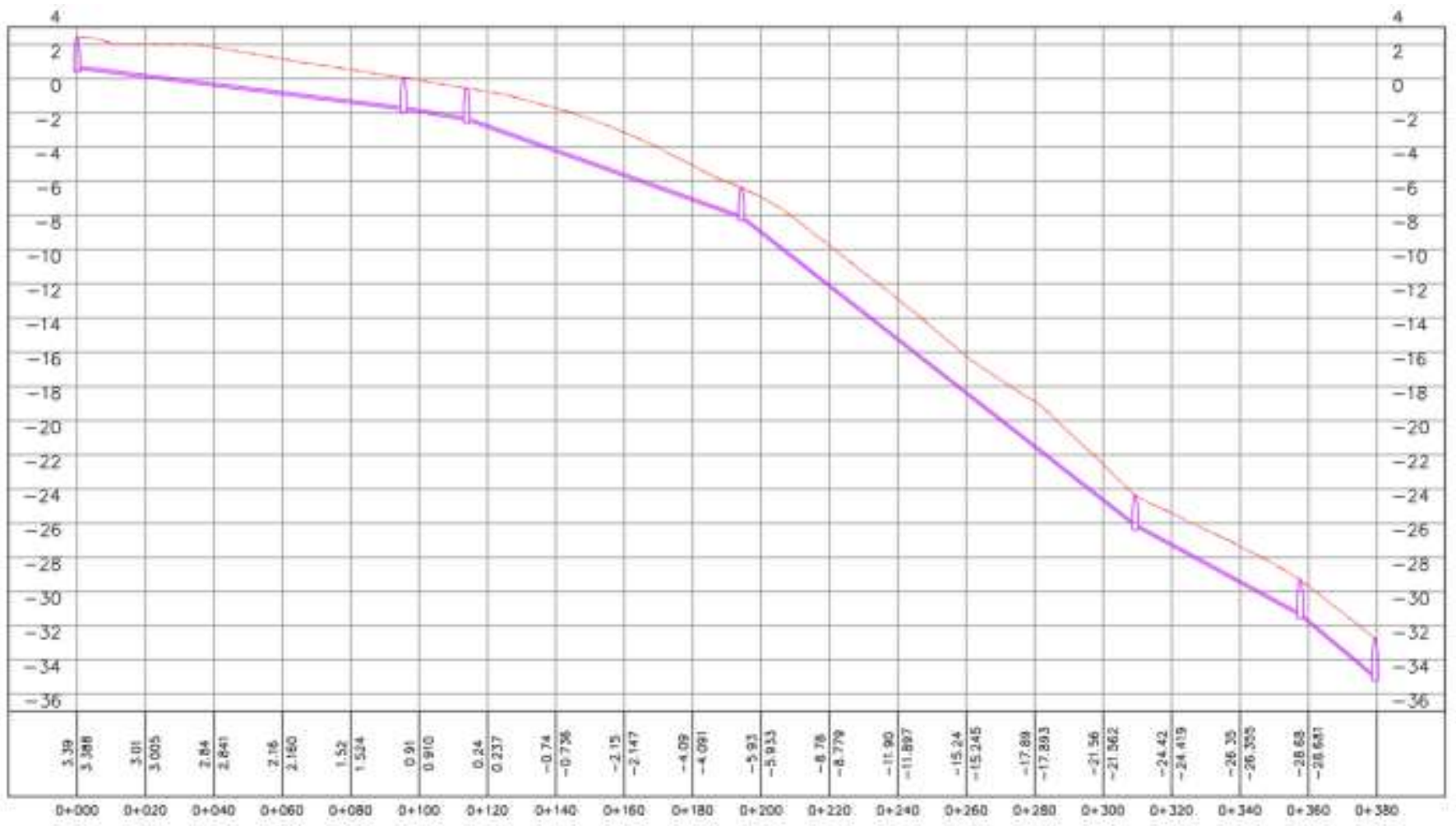

Fonte: Autores.

A Tabela 1, a qual é gerada automática pelo software, possui informações dos PVs, sendo que na primeira coluna está o nome da estrutura, na segunda tem detalhes como o diâmetro, a cota superior (RIM), a inferior (SUMP) e a cota (INV IN / INV OUT) na qual entram ou saem os tubos. As demais colunas representam os tubos que entram e que saem no PV.

Tabela 1 - Tabela com detalhes e quantitativo das estruturas do trecho 1 da rede coletora de esgoto.

\begin{tabular}{|c|c|c|c|}
\hline $\begin{array}{l}\text { Nome da } \\
\text { estrutura }\end{array}$ & Detalhes & Tubos que chegam & Tubos que saem \\
\hline \multirow{4}{*}{ PV1 } & $1500 \mathrm{~mm}$ & & \multirow{4}{*}{ PV1 - PV2, $150 \mathrm{~mm}$ PVC INV OUT =1,58 } \\
\hline & $\mathrm{RIM}=3,39$ & & \\
\hline & $\mathrm{SUMP}=1,57$ & & \\
\hline & INV OUT $=1,580$ & & \\
\hline \multirow{5}{*}{ PV2 } & $1500 \mathrm{~mm}$ & \multirow{5}{*}{$\begin{array}{c}\text { PV1 - PV2, } 150 \mathrm{~mm} \text { PVC INV } \\
\text { IN =-0,81 }\end{array}$} & \multirow{5}{*}{ PV2 - PV3, $150 \mathrm{~mm}$ PVC INV OUT =-0,81 } \\
\hline & $\mathrm{RIM}=1,06$ & & \\
\hline & SUMP $=-0,82$ & & \\
\hline & INV IN $=-0,814$ & & \\
\hline & INV OUT $=-0,814$ & & \\
\hline \multirow{5}{*}{ PV3 } & $1500 \mathrm{~mm}$ & \multirow{5}{*}{$\begin{array}{c}\text { PV2 - PV3, } 150 \mathrm{~mm} \text { PVC INV IN } \\
=-1,43\end{array}$} & \multirow{5}{*}{ PV3 - PV4, 150 mm PVC INV OUT =-1,43 } \\
\hline & $\mathrm{RIM}=0,44$ & & \\
\hline & SUMP $=-1,44$ & & \\
\hline & INV IN $=-1,430$ & & \\
\hline & INV OUT $=-1,430$ & & \\
\hline
\end{tabular}


Tabela 1 - Tabela com detalhes e quantitativo das estruturas do trecho 1 da rede coletora de esgoto (continuação)

\begin{tabular}{|c|c|c|c|}
\hline $\begin{array}{l}\text { Nome da } \\
\text { estrutura }\end{array}$ & Detalhes & Tubos que chegam & Tubos que saem \\
\hline PV4 & $\begin{array}{l}1500 \mathrm{~mm} \\
\text { RIM = -5,38 } \\
\text { SUMP = -7,17 } \\
\text { INV IN }=-7,160 \\
\text { INV OUT }=-7,160\end{array}$ & $\begin{array}{l}\text { PV3 - PV4, } 150 \mathrm{~mm} \text { PVC INV } \\
\text { IN }=-7,16\end{array}$ & PV4 - PV5, 150 mm PVC INV OUT =-7,16 \\
\hline PV5 & $\begin{array}{l}1500 \mathrm{~mm} \\
\text { RIM }=-23,41 \\
\text { SUMP }=-25,27 \\
\text { INV IN }=-25,200 \\
\text { INV OUT }=-25,200\end{array}$ & $\begin{array}{c}\text { PV4 - PV5, } 150 \mathrm{~mm} \text { PVC INV IN } \\
=-25,20\end{array}$ & PV5 - PV6, $150 \mathrm{~mm}$ PVC INV OUT =-25,20 \\
\hline PV6 & $\begin{array}{l}1500 \mathrm{~mm} \\
\text { RIM }=-28,33 \\
\text { SUMP }=-30,42 \\
\text { INV IN }=-30,420 \\
\text { INV OUT }=-30,420\end{array}$ & $\begin{array}{l}\text { PV5 - PV6, } 150 \mathrm{~mm} \text { PVC INV } \\
\text { IN }=-30,42\end{array}$ & PV6 - PV7, 150 mm PVC INV OUT =-30,42 \\
\hline PV7 & $\begin{array}{l}1500 \mathrm{~mm} \\
\text { RIM }=-31,76 \\
\text { SUMP }=-34,08 \\
\text { INV IN }=-34,075\end{array}$ & $\begin{array}{l}\text { PV6 - PV7, } 150 \mathrm{~mm} \text { PVC INV } \\
\text { IN =-34,08 }\end{array}$ & \\
\hline
\end{tabular}

Na Tabela 2, também gerada automaticamente pelo Civil 3D, estão apresentados os detalhes dos tubos, sendo que na primeira coluna está o nome da estrutura, na segunda o diâmetro da tubulação, na terceira o comprimento do trecho, na quarta a inclinação do trecho e por último está descrito o material.

Tabela 2 - Detalhes e quantitativos dos tubos do trecho 1 da rede coletora de esgoto.

\begin{tabular}{ccccc}
\hline Trecho & $\begin{array}{c}\text { Diâmetro } \\
(\mathrm{mm})\end{array}$ & $\begin{array}{c}\text { Comprimento } \\
(\mathrm{m})\end{array}$ & $\begin{array}{c}\text { Declividade } \\
(\%)\end{array}$ & Material \\
\hline PV1 - PV2 & 150 & 95,4 & 2,51 & \\
PV2 - PV3 & 150 & 18,5 & 3,33 & \\
PV3 - PV4 & 150 & 80,3 & 7,13 & PVC \\
PV4 - PV5 & 150 & 115,2 & 15,66 & \\
PV5 - PV6 & 150 & 48,2 & 10,83 & \\
PV6 - PV7 & 150 & 21,9 & 16,71 & \\
\hline \multicolumn{5}{c}{ Fonte: Autoria própria. Fonte: Autores. }
\end{tabular}

\section{Conclusão}

A utilização de um software BIM na área do saneamento é incipiente. Por isso, neste trabalho se adotou uma metodologia de representação no Autodesk Civil 3D de um projeto de sistema de esgoto. A metodologia utilizada foi eficiente e, por meio dela, pode-se modelar o projeto e obter o perfil da rede, além do modelo 3D que permite uma análise detalhada. 
Ademais, também foi possível obter, de forma automática, o detalhamento e o quantitativo do projeto.

Como sugestão para trabalhos futuros, recomenda-se que o Autodesk Civil 3D seja utilizado também para o dimensionamento da rede coletora de esgoto e não apenas para a sua representação, como realizado neste trabalho.

\section{Referências}

ABNT. (1986). NBR 9649: Projeto de redes coletoras de esgoto sanitário. Associação Brasileira de normas técnicas.

Augustine, A. \& Eldhouse, S. (2016). 3D Model for Highway Alignment. International Journal of Scientific Engineering and Research, 4 (3), 63 - 67.

Barbosa, E. F. (2017). Bim e saneamento - Soluções técnicas para a concepção de estudos e projetos de sistemas lineares de esgotamento sanitário e abastecimento de água. In: Congresso ABES - FENASAN.

Bradley, A., Li, H., Lark, R. \& Dunn, S. (2016). BIM for infrastructure: An overall review and constructor perspective. Automation in Construction, 71, 13952.

Cardoso, M. C. (2020). Autodesk civil 3D 2020 aplicações BIM para projetos de infraestrutura. Érica.

Cardoso, R. R., Masieiro, F. R. S., Souza, E. M. \& Matta, P. S. (2021). Aspectos construtivos no uso do BIM. Episteme Transversalis, $11(3), 264-277$.

Carmona, F. V. F. \& Carvalho, M. T. M. (2017). Caracterização da utilização do BIM no Distrito Federal. Ambiente Construído, 17(4), 385-401.

Checcucci, E. S. (2019). Teses e dissertações brasileiras sobre BIM: uma análise do período de 2013 a 2018 . PARC - Pesquisa em Arquitetura e Construção, $10,1-17$.

Correa, S. L. M., Siviero, L. F., Freitas, R. O., Correa, F. R. \& Santos, E. T. (2019). BIM para infraestrutura de transportes. In: Simpósio Brasileiro de Tecnologia da Informação e Comunicação na Construção.

Costin, A., Adibfar, A., Hu, Hanjin, H. \& Chen, S. S. (2018). Building Information Modeling (BIM) for transportation infrastructure: Literature review, applications, challenges and recommendations. Automation in Construction, 94, 257 - 281.

Coutinho, A. B., Moura, G. S. \& Teixeira, E. K. C. (2021). Compatibilização de um projeto arquitetônico e hidrossanitário utilizando a metodologia BIM. Research, Society and Development, 10(2), $1-13$.

Eastman, C., Teicholz, P., Sacks, R. \& Liston, K. (2008). BIM Handbook: a guide to building information modeling for owners, managers, designers, engineers and contractors. New Jersey: John Wiley e Sons.

Edmondson, V., Cerny, M., Lim, M., Gledson, B., Lockley, S. \& Woodward, J. (2018). A smart sewer asset information model to enable an "Internet of Things" for operational wasterwaer management. Automation in Construction, 91, 193 - 205.

Gomes, R. M. S., Abreu, A. A., Bauer, M. A., Nascimento, G. C. \& Vale, M. S. (2018). Modelagem de informações para a construção (BIM): ambientes colaborativos para gestão de projetos e obras na construção civil. Perspectivas On-line: Exatas \& Engenharia, 8(23), 48 - 62.

Huang, S. F., Chen, C. H. \& Dzeng R. J. (2011). Design of track alignment using building information modeling. American Society of Civil Engeneering $(A S C E), 137,1-11$.

Kim, H., Orr, K., Shen, Z., Hyounseok, L., Ju, K. \& Choi, W. (2014). Highway alignment construction comparison using object-oriented 3D visualization modeling. Journal Construction Engeneering Management. 10, 1 - 13.

Li, N., Li, J., Peng, J., Cui, H. \& Wu, Z. (2018). A review of currently applied building information modeling tools of constructions in China. Elsevier, 201, $358-368$.

Maína, J. (2018). CAD and BIM in architecture education: awareness, proficiency and advantages from the student perspective. Gazi University Journal of Science Part B: Art Humanities Design and Planning, 6(4), 167-178.

Maranhão, T. A. \& Andrade, C. V. S. (2021). Estudo comparativo entre tecnologias de tratamento segmentado para reutilização de águas servidas e esgoto. Brazilian Journal of Development, 7 (6), 56691-56700.

Rodrigues, G. P. W.; Farias, G. M.; Costa, L. H. M. \& Castro, M. A. H. (2020). Otimização do traçado de redes coletoras de esgoto sanitário via algoritmo genético. Revista DAE, 68 (222), 164-177.

Salomão, P. E. A., Costa, N. C., Gouvêa, D. L., Oliveira, A. N. S. \& Silva, A. J. M. (2019). Modelagem e compatibilização de projetos de uma residência Minha Casa Minha Vida em software de plataforma BIM. Research, Society and Development, 8(8), $1-23$.

Santos, D. M., Piacente, F. J., Neves, J. M. S. \& Azevedo, M. M. (2021). Estudo comparativo da implementação da Modelagem da Informação da construção em obras públicas no Brasil e no Reino Unido. Research, Society and Development, 10(1), $1-13$.

Santos, D. M. \& Piacente, F. J. (2021). Indústria 4.0: Building Information Modelling no Custo de Construção Pública. Research, Society and Development, $10(1), 1-8$.

Smith, P. (2014). BIM Implementation - Global Strategies. Procedia Engineering. 85, 482-492. 
Research, Society and Development, v. 10, n. 15, e343101522860, 2021

(CC BY 4.0) | ISSN 2525-3409 | DOI: http://dx.doi.org/10.33448/rsd-v10i15.22860

Torres, X. E. L. (2020). BIM no planejamento de obras de infraestrutura de drenagem, São Paulo - SP (Dissertação de mestrado). Escola Politécnica, Universidade de São Paulo, São Paulo. 